\title{
Posicionamientos del pensamiento con respecto a la objetividad en la Enciclopedia de las Ciencias Filosóficas de Guillermo Federico Hegel
}

\author{
Positionings of the thought with regard to the objectivity in the Encyclopedy of the Sciences \\ Philosophical of Guillermo Federico Hegel \\ Rodolfo Carcía Aguilar ${ }^{1}$
}

Recibido: 20/1/2016 / Aprobado: 12/4/2016

\section{Resumen}

\begin{abstract}
El presente trabajo es un resumen sobre el tema "Tres posicionamientos del pensamiento con respecto a la objetividad" que Guillermo Federico Hegel desarrolla en su obra La Enciclopedia de las Ciencias Filosóficas, previo a iniciar el tema de la Lógica. La idea de exponer dicho tema, haciendo énfasis en el aspecto metodológico, es con el fin de preparar el camino conceptual para desarrollar en un futuro el tema de la lógica dialéctica.
\end{abstract}

Palabras claves: objetividad, lógica dialéctica, metafísica, concepto, Hegel, Filosofía Crítica.

\section{Abstract}

This paper is a summary of "Three positions of thought with regard to objectivity" that William Friedrich Hegel develops in his book Encyclopedia of the Philosophical Sciences, before beginning with the subject of Logic. The idea of presenting this summary, highlighting methodological aspects, is to prepare a conceptual way to address dialectical logic in the future.

Keywords: objectivity, dialectical logic, metaphysics, concept, Hegel, Philosophy Criticism.

\section{Introducción}

El presente trabajo es un resumen del apartado "Concepto Previo", de la primera parte de la lógica, según la tercera edición de la Enciclopedia de las Ciencias Filosóficas, de acuerdo con la exposición del autor en lo referente a la metodología de las filosofías anteriores, que preparan el camino para un nuevo acomodo conceptual: Lógica Dialéctica.

En un primer momento se expondrá la posición del autor con respecto a su lógica, luego, se expondrán las posiciones anteriores, criticadas, por el autor con el fin de extraer lo positivo en el metodológico.

Las filosofías anteriores caracterizadas por la apropiación de la objetividad, en orden de aparición son: la Metafísica, el Empirismo, la Filosofía Crítica y el Saber Inmediato. La crítica realizada es que dichas concepciones filosóficas estructuran su universo conceptual a partir de su propia lógica, a partir de sus propias determinaciones conceptuales, lo que incurre en su universalización, donde se consideran como referencia de toda la realidad, por lo cual se pierde de vista el papel de la realidad como criterio de verdad; el criterio de la verdad es la totalidad. Es decir, construyen su mundo conceptual a partir de su propio en sí, o sí logran trascender al en sí lo cosifican y lo universalizan como punto de referencia conceptual.

¿Qué es lo que aporta el autor al desarrollo de la filosofía que lo hace tan llamativo? ¿Qué es lo que aporta en su obra a la Lógica? ¿Cuál cambio de actitud se nota en el método hegeliano? La respuesta a estas preguntas está en la misma exposición, en

${ }^{1}$ Licenciado en Filosofia y Derecho. Profesor de Filosofía del Sistema de Educación General, Universidad de Costa Rica, Sede de Occidente. Correo electrónico: rga3cr@yahoo.com 
cuanto que a partir de las deficiencias señaladas a la metodología de las filosofías anteriores se puede ir vislumbrado el nuevo espacio metodológico, conceptual y lógico.

Hans Georg Gadamer grafica la nueva actitud filosófica perfeccionada por Hegel, cuando dice que esta “(...) trata de una progresión inmanente, que no pretende partir de ninguna tesis impuesta, sino más bien seguir el automovimiento de los conceptos, y exponer, prescindiendo por entero de toda transición designada desde fuera, la consecuencia inmanente del pensamiento en continua progresión" (Gadamer, 1981: 1)².

\section{Desarrollo}

Hegel inicia el apartado con una idea que será la guía de toda la exposición, y sobre la cual se basa el presente trabajo: "La lógica es la ciencia de la idea pura, esto es, de la idea en el elemento abstracto del pensar" (Hegel, 2010, pág 125). La lógica es la ciencia del pensamiento, de sus determinaciones y de sus elementos, donde está la idea en cuanto lógica; la idea se entenderá como la totalidad desarrollada a sí misma en sus determinaciones y leyes propias del pensamiento.

La lógica no tiene que ver nada con las intuiciones ni con representaciones sensibles abstractas, sino que en este nuestro caso, y dentro de la lógica dialéctica, tiene que ver con abstracciones “puras”. Así, el contenido de la lógica es el propio pensamiento y sus determinaciones corrientes, lo elemental: ser, nada, determinación, magnitud, seren-sí, ser-para-sí, uno, muchos, lo uno, lo múltiple, entre otras determinaciones.
El pensar en cuanto actividad es lo universal activo; representado como sujeto es lo pensante y la expresión simple del sujeto existente es yo. El yo es la existencia de la universalidad totalmente abstracta: lo abstractamente libre. Hay una diferencia fundamental entre el representar y el entendimiento, a saber: el representar tiene como su contenido lo sensible, bajo la determinación de lo mío, el contenido está en mí. Además tiene contenidos materiales procedentes del pensamiento autoconsciente, como las representaciones de lo justo, lo ético y lo religioso. El entendimiento, por su parte, se distingue de la representación en que este sienta relaciones de necesidad entre las determinaciones. La filosofía transforma representaciones en pensamientos, pero además transforma los meros pensamientos en concepto.

El pensar es la parte activa en relación con los objetos, el producto de la actividad del pensar es lo universal, contiene lo esencial, lo interior, lo verdadero. La reflexión cambia el contenido de la representación, de forma que se llega a la verdadera naturaleza del objeto. Con la reflexión aparece la verdadera naturaleza de la cosa; este pensar es la propia actividad del sujeto. Dentro de esta concepción, los pensamientos se pueden llamar objetivos si contemplan las formas de la lógica usual, y que se consideran meras formas del pensar consciente. Las relaciones entre concepto, juicio y silogismo con otras como la causalidad sólo se contemplan dentro de la misma lógica.

\footnotetext{
${ }^{2}$ Con respecto a esta temática, sirve también la referencia sobre los mundos contradictorios de los cuales parte el autor y que nos permite entender cuál es la síntesis que propone con su exposición, y cuál es el contexto que le exige dicha reflexión: "Hegel escribe que el hombre "tiene que vivir en dos mundos que se contradicen, de tal modo que también la conciencia se debate en esta contradicción; arrojada de un lado a otro, es incapaz de satisfacerse aquí y allá. En efecto, por una parte, vemos al hombre aprisionado en la actualidad ordinaria y a lo temporal terrestre, abrumado por la necesidad y la miseria, amenazado por la naturaleza, comprometido en la materia, en los objetivos sencillos y el goce, dominado por los instintos naturales y sus pasiones. Por otra parte, se eleva a las ideas eternas, a un reino de pensamiento y libertad; se asigna como Desarrollo". (Hegel citado por Garaudy, 1973: 13).
} 
(...) también hay que observar provisionalmente que al intentar el pensamiento hacerse con un concepto de las cosas, éste no puede consistir en determinaciones y relaciones que sean extrañas y exteriores a la cosa. Reflexionar (...) conduce a lo universal de las cosas; y lo universal es precisamente uno de los momentos del concepto (Hegel, 2010: 131).

La finitud de las determinaciones del pensamiento se deben ver en un doble aspecto: que las determinaciones son sólo subjetivas y retienen la oposición respecto de lo objetivo, y que estas determinaciones en cuanto contenido limitado en general permanecen en oposición a lo absoluto. Lo anterior refleja el pensamiento del autor, en una exposición lineal, ahora se verán los antecedentes de dicha posición, que el autor supera con su aporte.

Por eso es necesario considerar los posicionamientos del pensamiento con respecto a la objetividad para explicar el puesto que tienen la lógica en el sistema hegeliano. Es esta temática la que específicamente nos permitirá una propedéutica al tema y tratamiento de lo que es la lógica.

\section{La Metafísica}

La metafísica es el primer posicionamiento del pensamiento respecto de la objetividad, es el proceder ingenuo que, sin tener conciencia de la oposición del pensar dentro de sí y frente a sí, incluye la convicción que mediante la reflexión se conoce la verdad, en otras palabras, la conciencia conoce lo que son los objetos verdaderamente.

Con esta fe, el pensamiento se dirige directamente a los objetos, reproduce desde sí mismo el contenido de las sensaciones e intuiciones convertido en contenido del pensamiento, $y$ con él se satisface como contenido propio de la verdad (Hegel, 2010: 133).

Hegel ubica esta filosofía en lo que él llama metafísica anterior, en toda la producción filosófica pre-kantiana, fue la sencilla visión que tuvo el entendimiento de los objetos de la razón. Esta metafísica consideraba las determinaciones del pensamiento como determinaciones fundamentales de las cosas, lo que es se conoce como tal al pensarlo, y se consideraban aptas para ser predicadas de lo verdadero. Supone en general que el conocimiento de lo absoluto puede tener lugar al oponerle predicados sin investigar a las determinaciones del entendimiento.

En esta metafísica no se investigaba si los predicados son algo verdadero en sí o para sí, ni se investigaba la forma del juicio. Por ejemplo: Dios existe, Dios es eterno, el alma es simple, la cosa es una, etc., los predicados son de contenido limitado, y se toman desde fuera del sujeto.

Los objetos de esta metafísica eran totalidades que pertenecían en sí y para sí a la razón, o sea, al pensamiento de lo universal concreto en sí mismo, los tomaba de la representación, les aplicaba las determinaciones del entendimiento y las determinaciones puestas como base y como si fueran sujetos dados, sujetos acabados, y solo la representación tenía el poder, la lógica, la regla, para decidir sí los predicados eran o no eran convenientes y suficientes. Las representaciones del alma, mundo, Dios y cualquier temática teológica parecen garantizar al pensamiento, por de pronto, un asidero firme.

Esta metafísica llegó al dogmatismo porque según la naturaleza de las determinaciones finitas, aceptó que entre dos afirmaciones opuestas una tenía que ser verdadera y la otra falsa. Tres ejemplos de dicho posicionamiento del pensamiento respecto de la objetividad serían la Ontología, la Psicología Racional, la Cosmología y la Teología Natural o Racional.

En la Ontología o Doctrina de las Determinaciones Abstractas de la Esencia esta se ve obligada a enumerar las determinacines finitas de manera empírica y contingente, su contenido se fundamenta en la afirmación de que con tal o cual palabra se piensa algo, la rectitud del análisis coincidiría con el uso linguistico, pero no a la verdad y necesidad de esas determinaciones en sí y para sí. Aquí la no verdad dependería de la contradicción que se encuentre entre el sujeto de la representación 
y el concepto que debería predicarse de él: "Por consiguiente, sí la verdad no fuese nada más que la ausencia de contradicción, lo primero que se debería investigar en todo concepto sería si no contiene de suyo una tal contradicción interna" (Hegel, 2010: 137).

La situación de la Psicología Racional es que se refiere a la naturaleza metafísica del alma y trata al espíritu como una cosa. En el caso de la Cosmología, esta trata del mundo, de su contingencia, necesidad, eternidad, limitación en el espacio y el tiempo, de las leyes de sus cambios y trataba además de la libertad del ser humano y el origen del mal. Se hablaba de oposiciones absolutas: contingencia y necesidad; causa eficiente y causa final; esencia y fenómeno; libertad y necesidad; bien y mal, entre otras.

En la Teología Natural o Racional se trataba el concepto de Dios y las pruebas de su existencia. Aquí es interesante la cuestión de cuáles predicados le convienen o no a lo que nos representamos como Dios. Al concepto de Dios sólo le queda al final, la abstracción vacía de la esencia que es indeterminada. En la demostración de la existencia de Dios se ve la dificultad del paso de lo finito a lo infinito, Dios se determina como sustancia inmediata del mundo o como un objeto frente al sujeto como algo finito.

Los atributos de Dios se hunden en el concepto abstracto de la realidad pura, de una esencia indeterminada; el mundo finito aparece como un ser verdadero y Dios frente a él, entonces, la representación interpone distintas relaciones entre Dios y el mundo que son determinadas como atributos, que son, al fin, de naturaleza finita, en cuanto a relaciones finitas: bueno, poderoso, sabio. Pero esos atributos deben ser a la vez infinitos, lo cual es una contradicción, esto se soluciona al llevar el atributo a lo carente de determinación mediante un aumento cuantitativo, con lo que el atributo sobre Dios es aniquilado y reducido a la mera palabra.

\section{El empirismo}

El empirismo marca el segundo posicionamiento del pensamiento respecto a la objetividad, y nace de la necesidad de buscar un contenido concreto frente a las teorías abstractas del entendimiento, y una exigencia de demostrar todo dentro del campo de la determinaciones finitas: es buscar lo verdadero en la experiencia. El empirismo tiene como fuente de acreditación de sus definiciones, suposiciones y contenidos más determinados, las representaciones, es decir, al contenido procedente de la experiencia. Aquí la percepción singular es distinta de la experiencia, y esta posición eleva el contenido perteneciente a la percepción, al sentimiento y la intuición, a la forma de representaciones, esto son principios, leyes universales.

Las determinaciones universales no deben tener otro significado o validez que no sea el dado por la percepción, y no deben tener una justificación fuera del mismo fenómeno. Lo positivo de este posicionamiento del pensamiento con respecto a la objetividad es que el conocimiento empírico tiene su propio presente inmediato y su certeza.

Es en el empirismo donde se encuentra, en un primer momento, el principio de que lo que es verdadero tiene que estar en la realidad efectiva y ahí tiene que estar disponible para la percepción. Esta posición es contrapuesta al deber ser que desprecia la realidad efectiva y el presente, pues se vale de un más allá, que no puede tener otro origen y existencia que el entendimiento subjetivo.

Por otro lado, el Principio de Libertad en el empirismo, consiste en que el ser humano debe ver por sí mismo aquello que ha de tener por válido en su saber estando presente. En este sentido, el empirismo niega lo suprasensible puesto que su conocimiento se limita a lo finito, pero siempre permitiendo la abstracción y la universalidad formales.

El error del empirismo consiste en que utiliza categorías metafísicas y saca conclusiones utilizando el silogismo, pero ignora que utiliza dichas categorías de una forma acrítica "Una consecuencia importante de lo dicho es que, bajo este modo empírico, las 
determinaciones y leyes jurídicas o ética, así como el contenido de la religión aparecen como algo contingente y se abandona su objetividad y verdad íntima" (Hegel. 2010, 141).

\section{La filosofía crítica}

La filosofía crítica también acepta la experiencia como única base del conocimiento, pero para el conocimiento de los fenómenos, no así el conocimiento de verdades. Es parte primera de la distinción entre los elementos que se encuentran en la experiencia, la materia sensible, y sus referencias universales, las cuales ya no proceden de lo empírico, sino que pertenecen a la espontaneidad del pensar a priori.

Las determinaciones del pensamiento o conceptos del entendimiento agotan la objetividad de los conocimientos de la experiencia, contienen referencias y se forman mediante juicios sintéticos $a$ priori: relaciones originarias de opuestos. Esto quiere decir que en el conocimiento se encuentran las determinaciones de la universalidad y la necesidad.

La filosofía crítica investiga en primera instancia el valor de los conceptos del entendimiento utilizados en la metafísica en lo referente a la oposición entre subjetivad y objetivad en general. Objetividad sería el elemento de la universalidad y necesidad, o las propias determinaciones del pensamiento, lo apriorístico. La filosofía crítica amplía la oposición de tal manera que termina predominando el lado de la subjetividad, y frente a ella, no queda nada más que la cosa en sí.

formas de lo apriorístico, del pensar en cuanto actividad subjetiva sin atender a la objetividad, se dan da en relación con la facultad teorética, el conocimiento en cuanto tal. Esta forma tiene como fundamento determinado de los conceptos del entendimiento la identidad originaria del yo, la unidad transcendental de la autoconciencia. Las representaciones de la sensación y de la intuición son algo plural según su contenido y forma, esto por la exterioridad recíproca de la sensibilidad en sus formas de espacio y tiempo; representaciones que en cuanto formas de lo universal son ellas mismas a priori.
Es sabido que la filosofía kantiana se puso las cosas fáciles con el hallazgo de las categorías. Yo, la unidad de la autoconciencia, es algo enteramente abstracto e indeterminado; ¿cómo se llega, por tanto, a las determinaciones del yo, a las categorías? (Hegel, 2010, 144).

En la lógica tradicional se encuentran las diferentes clases de juicios ofrecidas de manera empírica. Juzgar es pensar un objeto determinado, las distintas formas de juzgar previamente catalogadas proporcionan las distintas determinaciones del pensamiento. Las determinaciones del pensamiento deben ser mostradas con su necesidad, o sea, deben ser deducidas de manera esencial.

Por medio de las categorías, la percepción se eleva a objetividad, a experiencia, pero estos conceptos que solo pertenecen a la conciencia subjetiva, condicionada por la materia dada, son vacíos y solo tienen su aplicación en la experiencia.

La cosa en sí (y bajo el término cosa se comprende también al espíritu, a Dios) expresa el objeto en la medida en que se abstrae de todo lo que éste es para la conciencia, de todas las determinaciones de la sensación, así como de todos los pensamientos determinados referidos a él (Hegel, 2010, 145).

Entonces, las categorías tienen su fuente en la unidad de la autoconciencia, y el conocimiento que se adquiere por su medio no contiene nada objetivo; lo objetivo que se le atribuye a las categorías es meramente subjetivo.

En la Filosofia Crítica se juzga la aplicación de las categorías a lo incondicionado según se dan en la metafísica, siguiendo el siguiente orden:

\section{III.1. Primer incondicionado: el alma}

III. 1.1. En la metafísica la conciencia se encuentra a sí misma como: un sujeto determinante, como algo singular o algo abstracto, como lo uno y lo mismo o identidad; y como ser pensante de todas las cosas fuera de mí. La metafísica ponía determinaciones del pensamiento en el lugar de 
determinaciones empíricas, por ejemplo: a.- el alma es sustancia, b.- la sustancia es simple, c.- la sustancia es idéntica a lo largo de los distintos tiempos de su existencia y d.- la sustancia está en relación con lo espacial. Este procedimiento tiene el defecto de que confunde determinaciones empíricas con categorías y concluye desde las determinaciones las categorías, lo cual es ilegítimo, pues pone las segundas en lugar de las primeras.

La crítica realizada a este proceder es que las determinaciones del pensamiento en general no se encuentran en la percepción, que lo empírico según su contenido y forma es distinto de la determinación del pensamiento. Es el caso del alma, sobre la base de las determinaciones que la conciencia permite experimentar sobre el alma, estas no son las mismas que el pensamiento produce al respecto.

La Filosofía Crítica hace consistir el conocer en general y el experimentar en el hecho de que las percepciones deben ser pensadas. Así, las determinaciones que pertenecientes primeramente al percibir deben ser transformadas en determinaciones del pensar. Cuando el pensamiento y el fenómeno no se corresponden adecuadamente, se opta por considerar como deficiente lo uno o lo otro; en el caso del idealismo kantiano, el defecto se achaca a el pensamiento, de modo que los pensamientos se consideran insuficientes.

III.1.2. La razón en su intento por conocer el mundo cae en antinomias, esto es en la afirmación de dos tesis contrapuestas sobre un mismo objeto, de tal manera que cada una de las tesis se afirma con la misma necesidad. La solución a dicha antinomia está en que la contradicción no recae sobre el objeto en y para sí, sino que es asunto de la razón cognoscente

\section{III.1.3. El tercer objeto de la razón es Dios.}

Dios deviene como un algo abstracto simple, cuya determinación es también simple, abstracta, el ser. La identidad abstracta y ser son los dos momentos cuya unión es el ideal de la razón. Esta unión propuesta por Kant se puede obtener por dos vías: se puede empezar por el ser y desde ahí pasar a lo abstracto del pensar o pasar de lo abstracto al ser. Si se inicia desde el ser, este se presenta como lo inmediato, un ser determinado de manera infinitamente múltiple, es mundo pleno, es una colección de infinitas contingencias en general, o se puede determinar como una colección de infinitos fines y de relaciones teleológicas.

Pensar este ser pleno significa quitarle la forma de las singularidades y contingencias y captarlo como un ser universal, necesario en y para sí, como ser activo que se determina con arreglo a fines universales, el cual es distinto de aquel ser primero, abstracto; significa captarlo como Dios (Hegel, 2010: 151).

La crítica realizada a esta vía es que las percepciones y agregado, el mundo, no muestran tener en sí la universalidad que el pensamiento alcanza purificando el contenido, así, esta universalidad no es justificada por la representación empírica del mundo. El ascenso de pensamiento a Dios desde la vía de la representación empírica del mundo, es improcedente pues no se puede extraer de las percepciones lo universal y necesario tal y como se ha dicho anteriormente.

Porque el ser humano es un ser que piensa, nunca el sano entendimiento humano, como tampoco la filosofía, se dejarían convencer de que para elevarse a Dios se tenga que partir solamente de y desde la intuición empírica del mundo (Hegel, 2010: 151).

La elevación del pensamiento sobre lo sensible, o sea, la salida del pensamiento por encima de lo finito hasta el infinito es un salto, un salto que rompe la serie de lo finito; este tránsito es sólo pensar. Si el salto se presenta en forma de silogismos, al estilo de las pruebas de la existencia de Dios, el punto de partida es la contemplación del mundo, presentada como un fundamento firme. La referencia desde el mundo, punto de partida, hasta Dios, punto de llegada, se representa como afirmativa, como una conclusión a partir de algo que es y permanece a otro algo que también es. 
Un gran error consistiría en querer conocer la naturaleza del pensar sólo a partir de esta forma propia del entendimiento. Por el contrario, pensar el mundo empírico significa cambiar la forma empírica transformándola en un universal. Así, el pensamiento ejerce una actividad negativa sobre el fundamento, la materia percibida, cuando se determina por la universalidad, esto es, no se queda con la primera figura empírica.

Las pruebas metafísicas de la existencia de Dios son interpretaciones y descripciones deficientes de la elevación del espíritu desde el mundo a Dios, porque no expresan el momento de negación que se contiene en esa elevación o, mejor dicho, no lo ponen de relieve (...) (Hegel, 2010: 152).

El sentido de la elevación del espíritu consiste en atribuir al mundo un ser que solo es apariencia, no es el verdadero ser, no es verdad absoluta, sino que la verdad absoluta se encuentra en Dios, más allá del fenómeno, sólo Dios sería el verdadero ser. Se entiende el pensar de una manera subjetiva, y su determinación es la universalidad abstracta, la identidad formal, el pensar es lo opuesto a la verdad en tanto que universalidad concreta en sí misma ${ }^{3}$.

\section{El saber inmediato}

Este posicionamiento consiste en aprender el pensamiento como actividad de lo particular únicamente, donde se tienen las categorías como producto del pensamiento, y se consideran determinaciones limitadas, formas de lo condicionado. Aquí lo infinito, lo verdadero, no es, puesto que no se da el paso hacia lo verdadero. Estas determinaciones limitadas son los conceptos. Concebir un objeto es captarlo bajo la forma de algo condicionado y mediado, así, cuando el objeto es lo verdadero, lo infinito e incondicionado, concebirlo significa cambiarlo a lo condicionado, de esta manera, en vez de captar lo verdadero pensándolo, se convierte en no verdadero.

El conocimiento se comprende como conocimiento de lo finito, como un avanzar del pensamiento a través de series de condicionados a condicionado, en las que cada concepto que es condición es a su vez un condicionado: se trata de condiciones condicionadas. Lo anterior quiere decir que esta forma de concebir la realidad a partir del concepto inmoviliza la realidad, la detiene, con lo cual se logra una mejor manipulación de lo estático, pues siempre está ahí, a disposición del sujeto conocedor o cosificador.

Se afirma de igual manera que la verdad es para el espíritu, solo la razón es lo que constituye al ser humano y es el saber acerca de Dios. Como el saber mediado se limita a los contenidos finitos, entonces la razón es saber inmediato, es fe. Saber se contrapone frecuentemente a creer, y la creencia, determinada como saber inmediato, resulta reconocida como saber. Como hecho empírico, se considera que lo que uno cree está en la conciencia y que así sabe de ello, y lo considera como algo cierto. El pensar se contrapone al saber inmediato, al creer, y a la intuición. Creer se dice también de las cosas comunes, del presente sensible, así se dice que se cree en la existencia de las cosas sensibles.

Pero ahora hay que tomar creer e intuir también en sentido superior, como fe en Dios, como intuición intelectual de Dios. Es decir, que hay que abstraer precisamente de aquello que constituye la distinción entre intuir y creer por una parte, y pensamiento por otra (Hegel, 2010: 168).

\footnotetext{
${ }^{3}(. .$.$) existe en el criticismo kantiano la afirmación de un profundo humanismo: el hombre es raíz y fuente de todas las cosas.$ "Kant ubica la verdad en el hombre". Todo comienza con la libertad del hombre que afirma su poder sobre la naturaleza y la sociedad y que ya no acepta al Dios revelado, “dado", de las religiones positivas (Hegel, citado por Garaudy, 1973: 116).
} 
La fe en esta posición filosófica es la autoridad de la propia revelación subjetiva, se trata de un contenido indeterminado en sí mismo: se trata así de la seca abstracción de saber inmediato. En otras concepciones esta fe se conoce como inspiración, revelación del corazón, lo innato, entendimiento humano, sentido común, etc.; y todas estas formas tienen el mismo contenido: la inmediatez con que se encuentra un contenido en la conciencia lo convierte en principio "Sin embargo, la tesis en torno a la cual gira todo el interés de la filosofía moderna, se puede decir, fue también expresada por su fundador bajo el modo de inmediatez: cogito, ergo sum" (Hegel, 2010: 169).

Esta posición conlleva a varias determinaciones, por ejemplo, lo que yo encuentro en mi conciencia, entonces, es algo que hay que encontrar en la conciencia de todos los seres humanos, a la vez que se toma como la naturaleza misma de la conciencia. Al encontrar un contenido en mi conciencia, junto con la certeza de su verdad, ella pertenece a la naturaleza misma del espíritu, no me pertenece sólo como sujeto.

En el saber inmediato se sabe que Dios es, pero, no se sabe qué es Dios, puesto que lo último es ya conocimiento. Dios en esta posición, como objeto de religión, es general, es indeterminado, y la religión reducida al mínimo en cuanto contenido.

La filosofía cartesiana parte de una presunción no demostrada y aceptada como indemostrable a un saber más desarrollado, dando origen a las ciencias de los tiempos modernos.

Como momento de superación sobre las posiciones vistas, el autor asoma por primera vez lo que será la lógica dialéctica, al señalar algunas generalidades, con lo cual termina esta parte introductoria de la pequeña lógica. Así, lo dialéctico es escepticismo, es contener la negación como resultado. La dialéctica se considera como una habilidad que puede producir una confusión en determinados conceptos y una mera apariencia entre ellos, de modo que lo nulo no es la determinación, sino la apariencia de contradicción, y lo que el entendimiento capta es más bien lo verdadero ${ }^{4}$, "Pero en su determinación propia, la dialéctica es más bien la propia y verdadera naturaleza de las determinaciones el entendimiento, de las cosas y de lo finito en general" (Hegel, 2010, 183).

La dialéctica es el superar inmanente en el cual se expone la unilateralidad y limitaciones de las determinaciones del entendimiento, a modo de negación. Es un proceder científico hacia adelante confiriendo conexión inmanente al contenido de la ciencia. Lo racional-positivo aprehende de la unidad de las determinaciones en su oposición, o sea, lo afirmativo que contiene la disolución de las determinaciones.

El resultado de la dialéctica es positivo, por cuanto tiene un contenido determinado, su resultado es la negación de la determinación tenidas como ciertas, en las otras opciones el contenido es abstracto y vacío. El resultado racional es algo concreto porque se trata de una unidad de determinaciones distintas.

\section{Conclusiones}

Es interesante observar como el autor a partir de la relación objeto-sujeto y sujeto-objeto logra evidenciar las distintas posturas filosóficas que lo antecedieron, donde las considera partes integrantes de una totalidad que es única en su esencia, pues al ser racional, es universal, y es la manifestación más perfecta de la presencia del ser humano en este mundo. Como característica de estas posiciones está el hecho del apoderamiento que tiene uno de los elementos dichos sobre el otro, generando una visión unilateral, única, particular de la relación objeto-sujeto-sujeto-objeto.

\footnotetext{
${ }^{4}$ Todo conocimiento real debe pasar por tres momentos: el de lo inmediato y de lo universal abstracto, luego el de la negación, que es reflexión, mediación, y el de la totalidad concreta, de lo universal concreto, es decir, del resultado que conserva y contiene en él el momento de la negación, de la reflexión, de la mediación. (Garaudy, 1973: 166)
} 
¿Cuál es entonces la verdadera posición?, la respuesta es: todas. Donde la lógica dialéctica lo que hará es dinamizar lo ya hecho por el ser humano. Menospreciar una posición anterior sería eliminar una parte del todo, y lo que existe es el todo, o sea, el proceso, el sistema, lo absoluto.

Se está ante una posición conciliatoria filosófica, donde no se elimina nada, sino que se integra en una sola realidad conceptual toda la creación, la cultura, el progreso de la razón. No se puede ser futuristas sobre que pasará con la dialéctica, y el espíritu, puesto que el autor se ubica y muesrtra el presente, no especula sobre el deber ser, pues sacrificaría el presente, el ser.

El automovimiento del concepto propuesto por Hegel descansa en la absoluta mediación de la conciencia y su objeto. La conciencia prepara el elemento del saber puro, que en modo alguno es un saber de la totalidad del mundo sino un saber del saber. El objeto sabido no puede ser jamás separado del sujeto que sabe, lo que quiere decir que cuando está en su verdad es en la autoconciencia del saber absoluto, hay un automovimiento del concepto. En el fondo, es una propuesta de ser conscientes de que el ser hmano es lo que crea: la realidad, la cultura, y esta es desarrollo de su creación.

La apropiación de la objetividad es un paso necesario hacia el desarrollo de la autoconciencia que vuelve a sí misma. Es sólo un modo de ser del ser humano, dentro de un proceso de creación y por qué no de supervivencia conceptual.

\section{Bibliografía}

Abbagnano, N. (1974). Diccionario de Filosofía. Segunda edición, Traducción de Alfredo N. Galletti. México: Fondo de Cultura Económica.

Copleston, F. (1980). Historia de la filosofía. Tomo 7, Traducción de Victoria Campos. Barcelona: Editorial Ariel.

Gadamer, H. G. (1981). La dialéctica de Hegel. Segunda edición, Traducción de Manuel Garrido. Madrid: editorial Cátedra Teorema.

Garaudy, R. (1976). Dios ha muerto. Traducción de Alfredo Llanos. Buenos Aires: Ediciones siglo veinte.

Hegel, G. F. (1985). Fenomenología del Espíritu. Traducción de Wenceslao Roces. México: Fondo de Cultura Económica. Sexta reimpresión.

(2010). Enciclopedia de las ciencias filosóficas. Traducción de Ramón Valls Plana. España: Alianza editorial, cuarta reimpresión.

(1975). Principios de la Filosofía del Derecho o Derecho Natural y Ciencia Política. Traducción de Juan Luis Vermal. Buenos Aires: Editorial Sudamericana. 